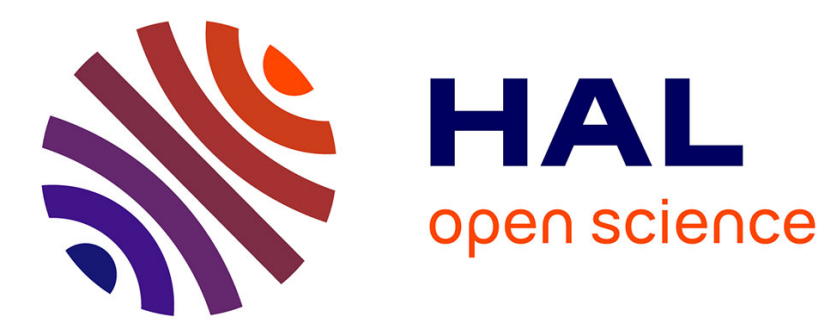

\title{
L'évitement scolaire et les classes moyennes à Paris
} Jean-Christophe François, Franck Poupeau

\section{To cite this version:}

Jean-Christophe François, Franck Poupeau. L'évitement scolaire et les classes moyennes à Paris. Education et Sociétés: Revue internationale de sociologie de l'éducation, 2004, 2 (4), pp.51 à 66. halshs-00160717

\section{HAL Id: halshs-00160717 https://shs.hal.science/halshs-00160717}

Submitted on 6 Jul 2007

HAL is a multi-disciplinary open access archive for the deposit and dissemination of scientific research documents, whether they are published or not. The documents may come from teaching and research institutions in France or abroad, or from public or private research centers.
L'archive ouverte pluridisciplinaire HAL, est destinée au dépôt et à la diffusion de documents scientifiques de niveau recherche, publiés ou non, émanant des établissements d'enseignement et de recherche français ou étrangers, des laboratoires publics ou privés. 


\title{
L'évitement scolaire et les classes moyennes à Paris
}

\author{
JEAN-ChristopHE FRANÇOIS \\ Université Paris VII, UMR 8504 Géographie-Cités \\ I 3 rue du Four, 75006 Paris
}

\section{FRANCK POUPEAU}

Centre de sociologie européenne, ESA 8035

54 boulevard Raspail, 75006 Paris

\begin{abstract}
Darfois décrites comme un milieu hétérogène regroupant aussi bien travailleurs indépendants que salariés, balayant un éventail de professions allant des fonctionnaires et ingénieurs aux petits patrons et boutiquiers, les "classes moyennes" n'en sont pas moins saisies sous un angle homogénéisant dont la dénomination tend à rabaisser la signification: leur position intermédiaire entre deux classes (dominantes et populaires, favorisées et défavorisées) les voue au désintérêt réservé aux positions ordinaires et banales, auxquelles renvoie le qualificatif moyen. Cette définition doublement négative exprime la difficulté à délimiter un groupe clairement identifiable — car s'il est vrai que "toute définition est négation", les classes moyennes sont avant tout ce que les autres classes sociales ne sont pas. La détermination logique ne suffit pourtant pas à donner une cohérence sociologique, dans la mesure où la définition des classes moyennes conduit moins à identifier un groupe social précis qu'à désigner le référent d'un ensemble de caractéristiques dépréciatives, dont la seule cohérence résiderait "dans la volonté de promotion sociale, dans la crainte des aléas de la conjoncture et dans l'exigence d'une protection de l'État contre le risque de remise en cause d'un statut chèrement acquis" (Berstein 2001, 13).
\end{abstract}

La reproduction de leur statut social dépendant de l'école davantage que pour les autres catégories sociales, les classes moyennes seraient celles qui chercheraient le plus à maximiser leurs investissements scolaires: elles se trouvent tout naturellement désignées comme les principales responsables des pratiques d'évitement scolaire, qui dans les taxinomies administratives sont regroupés sous le terme d'“évaporation" (Broccolichi \& van Zanten 
1997). Ce phénomène recouvre pourtant différents types de trajectoires qui ne sont pas forcément assimilables, depuis le recours à l'enseignement privé jusqu'au choix d'un établissement public hors du secteur attribué par la carte scolaire, notamment via une dérogation officielle (annexe I). Mais dans tous les cas, la position des classes moyennes semble les désigner comme les principales responsables de l'évitement, coupables du refus de mixité sociale que se plaisent toujours à dénoncer ceux qui en sont le plus préservés: en ce qui concerne le choix des établissements publics, elles seraient aussi les plus portées à adopter des stratégies de fuite de l'établissement du secteur (évitement négatif), afin de se mettre à distance des couches populaires.

Cet article s'attache à analyser les déterminants sociaux des pratiques d'évitement scolaire à l'entrée en sixième —en France, la première année de l'enseignement secondaire - au sein du secteur public à Paris. Le choix de ce terrain d'étude se justifie par la valeur exemplaire du cas parisien, où l'on peut observer des mécanismes qui ne seraient pas aussi visibles ailleurs. En effet, la forte densité de la population induit d'une part une forte densité du semis des établissements publics et privés. La centralité, d'autre part, renforce l'offre en induisant la localisation intra-muros d'établissements atypiques ou spécialisés; elle induit également une bonne accessibilité des établissements scolaires par les transports en commun (sur l'espace scolaire parisien, François, Mathian, Ribardière \& Saint-Julien 2002). Après avoir rappelé la division sociale de l'espace scolaire parisien et ses relations avec l'espace résidentiel, il s'agit de proposer une modélisation des comportements individuels qui précise l'importance relative des différentes variables susceptibles d'expliquer les pratiques d'évitement. Dans tous les modèles proposés, on cherche à expliquer les choix individuels des parents d'élèves en termes d'évitement selon des variables telles que l'appartenance sociale, l'âge scolaire et l'éventuelle interaction des deux. Dans un premier temps (modèle global), l'appartenance sociale est seulement approchée, puisque sont seulement mobilisées les quatre grandes catégories sociales en usage dans l'administration scolaire. Ce faisant, on se contente d'un dessin un peu grossier de "la classe moyenne", mais, en contrepartie, l'importance des effectifs dans chaque sous-population renforce la robustesse statistique du modèle. Dans un deuxième temps, on affine le découpage social de référence en vingt-quatre catégories professionnelles (PCS): pour chacune d'elles, on s'attache alors à modéliser les relations entre âge scolaire et évitement afin de voir si elles obéissent toutes aux mêmes mécanismes ou si elles expriment une certaine transformation des pratiques scolaires au sein des stratégies de reproduction des différents groupes sociaux. 


\section{Espace social et espace scolaire à Paris}

T 'évitement scolaire peut être mesuré par le décalage entre la population résidente et la population scolarisée dans les établissements publics. Des études consacrées aux effets de l'évitement scolaire sur la division sociale de l'espace scolaire de Paris intra-muros montrent que l'évitement est un révélateur de la différenciation des stratégies de scolarisation dans les différentes catégories sociales (François 2002). Cette différenciation s'inscrit en premier lieu dans les structures scolaires de l'espace parisien qui se caractérise par une grande diversité de l'offre et un profil social atypique: forte densité des catégories favorisées, forte (et ancienne) différenciation sociale de l'espace entre un ouest bourgeois et un nord-est populaire, même si ce dernier est marqué par un mouvement d'embourgeoisement récent. Les pratiques d'évitement y sont relativement stables et anciennes: la propension à demander une dérogation à l'entrée en sixième s'élève à $15 \%$ en 1992 , contre $16 \%$ en 2001. Toutefois, durant cette période, le taux de satisfaction des demandes a diminué fortement, une politique de sectorisation stricte ayant remplacé la politique d'assouplissement de la carte scolaire introduite à la fin des années 1980. Ce taux de satisfaction des dérogations à la carte scolaire atteint $49 \%$ en 2001, toutes catégories confondues, et la probabilité de voir une demande satisfaite est relativement indépendante de la catégorie sociale du demandeur -ce résultat contre-intuitif pouvant s'expliquer par la procédure mise en place par le rectorat de l'académie_- : pour chaque établissement, les demandes invoquant le même motif sont étudiées en bloc, selon les résultats disponibles, ce qui minimise l'efficacité des stratégies individuelles. Cependant, les taux d'évitement demeurent étroitement liés à la hiérarchie des catégories sociales: le filtrage social s'effectue surtout en amont, par une inégale propension des différentes catégories sociales à éviter, les plus favorisés évitant davantage. Ces différences sont plus marquées encore en ce qui concerne le recours au privé qu'en ce qui concerne les dérogations (tableau 1).

Alors que la propension à déroger est liée à l'appartenance sociale de l'élève, la carte des dérogations ne reproduit pas vraiment la carte des disparités sociales de l'espace scolaire parisien. Le pôle attractif constitué par la zone centre-ouest abrite des établissements qui ne polarisent pas seulement un grand nombre de dérogations importantes en leur faveur, mais connaissent en même temps des fuites importantes vers le privé. Les mouvements en cascade observables expriment alors le refus, par certaines familles de ces quartiers, de la mixité sociale (pourtant bien limitée) induite par l'arrivée, via les dérogations, d'élèves de quartiers moins favorisés. Cependant, malgré ces remous locaux, la division de l'espace scolaire parisien reste globalement stable: l'évitement a surtout pour effet d'accentuer les polarisations existantes, c'est-à-dire de renforcer les discontinuités entre le Paris favorisé et le Paris défavorisé (François 2002). 
Tableau I

Taux d'évitement en fonction de l'origine sociale*

\begin{tabular}{|l|c|c|c|c|c|}
\hline & Favorisés A & Favorisés B & Moyens & Défavorisés & $\begin{array}{c}\text { Toutes } \\
\text { catégories }\end{array}$ \\
\hline $\begin{array}{l}\text { Propension à choisir le privé au } \\
\text { moment de l'entrée en sixième }\end{array}$ & 0.09 & 0.08 & 0.07 & 0.02 & 0.06 \\
\hline $\begin{array}{l}\text { Propension à demander une } \\
\text { dérogation à la carte scolaire au } \\
\text { moment de l'entrée en sixième }\end{array}$ & 0.22 & 0.19 & 0.16 & 0.10 & 0.16 \\
\hline $\begin{array}{l}\text { Propension à éviter (formes } \\
\text { illicites non comprises) au } \\
\text { moment de l'entrée en sixième }\end{array}$ & 0.31 & 0.27 & 0.23 & 0.12 & 0.22 \\
\hline
\end{tabular}

* Les catégories utilisées par le ministère de l'Éducation nationale regroupent les professions suivantes:

— personne sans activité professionnelle, chômeur n'ayant jamais travaillé, retraité employé et ouvrier, ouvrier agricole, ouvrier non qualifié pour origine "défavorisée";

- retraité agriculteur exploitant, personnel service direct aux particuliers, employé de commerce, employé administratif d'entreprise, policier et militaire, agent de service de la fonction publique/ employé de service, chef d'entreprise de dix salariés ou plus, commerçant et assimilé, artisan, agriculteur exploitant pour origine "moyenne";

- retraité artisan/commerce/chef d'entreprise, contremaître, agent de maîtrise, technicien, profession intermédiaire administrative/commerciale d'entreprise, profession intermédiaire administrative de la fonction publique, clergé, religieux, profession intermédiaire de la santé/travail social, pour origine "favorisée B";

- retraité cadre/profession intermédiaire, ingénieur/cadre technique d’entreprise, cadre administratif et commercial d'entreprise, profession de l'information, des arts et du spectacle, professeur et assimilé, instituteur et assimilé, cadre de la fonction publique, profession libérale pour origine "favorisée A".

On peut donc s'interroger sur le rôle joué par les classes moyennes dans ces mécanismes d'accentuation des disparités sociales. Si elles regroupent, comme le veut l'opinion commune, les catégories qui recourent le plus aux stratégies d'évitement, il est alors possible de leur imputer une grande partie du décalage entre structures sociales et structures scolaires, quels que soient les motifs utilisés: désir de réussite scolaire incitant au placement dans un établissement réputé (évitement positif), ou bien fuite hors des établissements du secteur, pour des motifs tels que le rejet de la mixité sociale dans les établissements accueillant des publics populaires (évitement négatif). La distinction proposée ici entre évitements positif et négatif ne doit pas faire oublier que ces deux ordres de motivation ne sont ni toujours conscients, ni nécessairement exclusifs. On doit en outre rappeler que les résultats présentés ici correspondent à une première étape du travail qui n'intègre pas la localisation géographique des élèves: on ne prend donc pas en compte les caractéristiques des établissements de départ.

Les thèses précédemment évoquées sur l'évitement des classes moyennes posent ainsi plusieurs types de problèmes. Tout d'abord, elles tendent 
à considérer leurs pratiques indépendamment de celles des autres groupes sociaux. Or il n'est pas évident a priori qu'elles aient des pratiques de scolarisation spécifiques. On peut penser en effet que leurs pratiques peuvent être déterminées non seulement par un éventuel rejet des milieux populaires, mais aussi par la diffusion des modèles scolaires des couches les plus favorisées et les mieux dotées (culturellement et économiquement). De plus, l'analyse en termes de stratégies risque de montrer les classes moyennes comme un groupe, sinon une somme d'individus, animé(s) par des calculs rationnels dans un espace isotrope. Or, un grand nombre de perturbations sont susceptibles de venir secouer ce modèle idéal qui croise des facteurs géographiques et sociologiques: l'inégal accès à l'information, l'accessibilité physique (temps et coût du transport) ou encore les modes de "présentation de soi" des élèves et des parents face aux administrations scolaires (aspect physique, maîtrise du langage écrit et oral), dont les effets peuvent être traduits par une expression comme le "délit de sale gueule". On voit qu'il convient de tenir compte de l'ensemble de l'espace social (objet du présent article) et de l'espace géographique (objectif du programme de recherche dont il est le premier jalon, voir annexe I).

\section{La différenciation des destinées scolaires à Paris selon l'origine sociale et l'âge scolaire}

Dans le cadre d'une enquête sur les disparités scolaires en Île-de-France dans des lieux diversifiés de l'espace francilien une modélisation statistique des déterminants de l'évitement scolaire a été entreprise afin de cerner plus précisément les modalités du phénomène d'évitement scolaire (annexe I). On se propose ainsi d'expliquer la propension d'un élève du secteur public entrant en sixième à éviter son collège de secteur à la fois par son origine sociale (mesurée à la catégorie socioprofessionnelle de l'adulte de référence), et par l'écart éventuel entre son âge réel et l'âge officiel d'entrée en sixième, onze ans. La population concernée est celle des élèves entrant en sixième dans un collège public de Paris intra-muros en septembre 2000 (n=15000), qu'ils habitent ou non dans le secteur de l'établissement où ils entrent. Sont exclus de l'analyse les élèves qui proviennent d'un établissement privé parisien $(n=600)$ ou d'un établissement (public ou privé) de banlieue $(n=400)$, les élèves qui ont déménagé entre le CM2 et la sixième et ceux dont la PCS n'est pas connue.

On n'a retenu dans cette analyse exploratoire que la sous-population des élèves de nationalité française $(n=12000)$ pour essayer de comprendre les liens entre origine sociale, âge et évitement (tableau 2). Bien qu'il ne s'agisse ici que de l'évitement interne à l'enseignement public, les pratiques prises en 
compte ne se limitent pas aux dérogations officielles: la source utilisant les adresses des élèves figurant dans le fichier de l'établissement, une partie des "pratiques occultes" est intégrée. La variable d'origine sociale (PCS) comprend ici seulement quatre modalités, selon la nomenclature de l'Éducation nationale (précisée au tableau 1): "favorisés A", "favorisés B", "moyens", "défavorisés". La variable ÂGE est déclinée en trois modalités ("en avance”, "à l'heure", "en retard").

Tableau 2

Taux d'évitement par origine sociale et classe d'âge

\begin{tabular}{|l|c|c|c|c|}
\hline \multicolumn{1}{|c|}{ PCS } & $\begin{array}{c}\text { Taux d'évitement } \\
\text { global }\end{array}$ & \multicolumn{3}{c|}{ Taux d'évitement par classe d'âge } \\
\hline & & En avance & À l'heure & En retard \\
\hline Favorisés A & 39 & 41 & 40 & 25 \\
\hline Favorisés B & 33 & 50 & 34 & 23 \\
\hline Moyens & 31 & 36 & 33 & 25 \\
\hline Défavorisés & 31 & 29 & 34 & 29 \\
\hline Ensemble & 34 & 40 & 35 & 27 \\
\hline
\end{tabular}

Les taux d'évitement diffèrent d'une catégorie sociale à l'autre, mais somme toute moins que l'on pouvait s'y attendre: dans la catégorie "favorisés A", un peu moins de quatre élèves sur dix évitent contre un peu plus de trois élèves sur dix dans la catégorie "défavorisés". En revanche, l'introduction de l'âge scolaire montre des taux d'évitement beaucoup plus contrastés entre les catégories d'élèves. Sachant qu'un élève parisien quelconque a une "chance" sur trois d'éviter son établissement de secteur, on va tenter de vérifier si l'appartenance sociale et l'âge scolaire jouent un rôle significatif en la matière. Pour cela, on propose un modèle de l'évitement scolaire, désigné sous l'appellation de modèle "global" dans la mesure où il s'efforce d'intégrer les diverses dimensions du phénomène étudié.

\section{Un modèle "global" de l'évitement scolaire}

La construction d'un modèle global sur l'évitement se fait au moyen d'un modèle log-linéaire "logit" (annexe 2). Chaque élève est décrit par ses caractéristiques individuelles, à savoir son appartenance sociale (variable PCS, quatre modalités) et son âge scolaire (variable ÂGE, trois modalités). Le modèle est construit pour prévoir au mieux le comportement d'un élève (évitera-t-il ou non?) en fonction de ses caractéristiques individuelles. Le rôle des différentes variables explicatives est résumé dans le schéma suivant. 


\section{Schéma résumant l'action des différentes variables}

Effets de l'âge et de l'origine sociale sur l'évitement à l'entrée en $6^{\mathrm{e}}$

Élèves de nationalité française, rentrée 2000

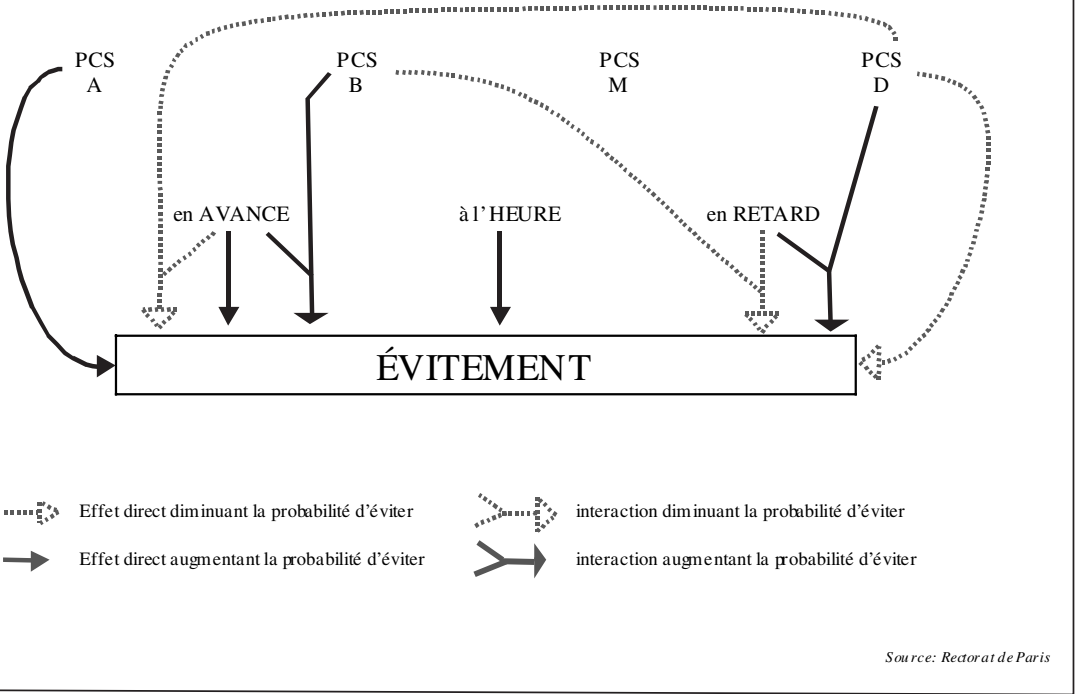

Si l'on considère la seule incidence de l'âge de l'élève, la probabilité de respecter la sectorisation est sensiblement réduite si l'élève est "en avance" et, dans une moindre mesure mais de manière encore significative, s'il est "à l'heure". En revanche, le fait d'être "en retard" diminue sensiblement la probabilité d'évitement. On sait par ailleurs que l'évitement à Paris est essentiellement un évitement "positif" (François 2002, Poupeau 2004), qui correspond plus au choix d'un collège censé être meilleur qu'à une volonté de fuir le collège de secteur. Dans cette mobilité, les élèves sans histoire et, à plus forte raison, ceux qui sont en situation de réussite réelle ou affichée (selon le critère de leur avance scolaire) ont un avantage certain: ils sont à la fois plus volontiers mobiles et plus efficaces dans leurs stratégies d'évitement. Inversement, la relative faiblesse numérique de l'évitement négatif permet de nuancer l'idée d'un évitement massif des classes moyennes désireuses de mettre les couches populaires à distance. Cette première conclusion débouche sur une hypothèse: il y a un marché des bons élèves réalisé par les établissements peu regardants sur la provenance géographique.

Indépendamment de l'âge scolaire, l'appartenance sociale, telle qu'elle est définie par les quatre modalités que distingue l'Éducation nationale, est nettement moins discriminante. L'appartenance aux catégories extrêmes constitue une variable significative dans le modèle : les élèves issus des catégo- 
ries "favorisées" évitent significativement plus et, réciproquement, les élèves “défavorisés" évitent significativement moins. En revanche, l'appartenance à la catégorie "moyenne" n'a quant à elle aucune incidence significative sur la probabilité d'éviter.

Les résultats les plus significatifs sont liés au croisement des deux variables précédentes. Si, une fois combinée à l'âge de l'élève, l'appartenance à la catégorie "moyenne" n'est pas non plus significative, c'est sans doute en raison de la diversité des comportements individuels induite par l'hétérogénéité sociale de ce groupe. En revanche, l'examen des interactions montre une différenciation très nette entre les élèves "favorisés A" et les "favorisés B". Pour les premiers, généralement dotés d'un fort capital culturel, le modèle indique que l'âge ne modifie pas la probabilité d'éviter. Pour les seconds, au contraire, l'âge jouerait un rôle discriminant, mais différent de celui qui s'applique à l'ensemble de la population étudiée. Pour que leur probabilité soit revue à la hausse, il faut nécessairement qu'ils soient "en avance". S'ils sont "en retard", ou même simplement "à l'heure", leurs chances d'éviter sont moindres. Toutefois cette différenciation sociale du rôle de l'âge scolaire dans l'évitement disparaît lorsque l'on construit un modèle prenant en compte le contexte géographique (piste de travail actuellement explorée dans le programme de recherche mentionné annexe 1). C'est le profil social de l'établissement de secteur, non l'origine sociale de l'élève lui-même, qui interagit alors avec l'âge scolaire pour influencer la probabilité d'éviter.

En revanche, les interactions impliquant les élèves de la catégorie "défavorisée" révèlent des résultats radicalement différents des autres catégories sociales. Pour un tel élève, les interactions avec l'avance et le retard scolaire sont significatives, mais jouent dans un sens opposé à ce que l'on observe pour l'ensemble de la population. En effet, toutes choses égales par ailleurs, un élève "défavorisé" voit sa probabilité d'éviter diminuer s'il est "en avance" et augmenter s'il est "en retard". Ce résultat ne peut manquer d'interroger.

Plusieurs hypothèses peuvent être émises. Du point de vue des élèves, l'évitement revêt des significations différenciées en fonction de l'origine sociale et des comportements associés face à l'avance ou au retard scolaire. Dans la catégorie "défavorisés", l'évitement semble plutôt constituer une réaction à des difficultés scolaires. S'il n'y a pas de retard scolaire en revanche, la sectorisation est respectée. On peut inversement supposer que les catégories les plus "favorisées" réagissent différemment aux difficultés scolaires, le recours au privé constituant une solution plus facilement offerte à ces familles (au moins en termes financiers), alors que les élèves issus de milieux populaires doivent se contenter de changer d'établissement public. Il existe toutefois un type d'établissement privé spécialisé dans l'accueil des élèves en situation d'échec issus des couches moyennes et, de façon croissante, des couches inférieures (Langouët \& Léger 1997). L'évitement peut alors être ana- 
lysé du point de vue des établissements, et des politiques de gestion des flux scolaires mises en place: on peut ici évoquer les pressions des établissements qui se débarrassent des "mauvais élèves" issus des catégories "défavorisées", en les orientant vers d'autres établissements, tout en conservant les "méritants" (généralement ceux qui sont "en avance"), notamment au moyen d'une offre d'options diversifiée.

Finalement, les catégories les plus "favorisées" se caractérisent bien par un évitement positif. Ce modèle des pratiques d'évitement au sein du secteur public à Paris semble indiquer que l'évitement est lié au désir de réussite scolaire. On a plus de mal en revanche à repérer un évitement négatif qui serait caractéristique d'une fuite des classes moyennes. En effet, le destin des élèves appartenant à la catégorie "moyenne" n'est pas influencé par les variables introduites, qu'elles soient considérées seules ou en interaction. Ce résultat peut indiquer que l'évitement de ces catégories passe davantage par le recours à l'enseignement privé, à condition d'admettre, compte tenu la rareté relative de cette pratique au moment de l'entrée en sixième (voir supra le tableau 1), que leur évitement commence précocement, dès l'école primaire. Mais il est possible que ce résultat soit en partie un produit de l'approximation liée à la définition des seules quatre catégories: c'est pourquoi une analyse détaillée des catégories socioprofessionnelles permet maintenant d'examiner les différenciations de pratiques de professions proches dans l'espace social et, en particulier, les transformations des stratégies de reproduction sociale.

\title{
La différenciation sociale des pratiques d'évitement
}

\begin{abstract}
Ain d'affiner l'analyse de l'effet différentiel de l'âge scolaire sur les prati- ques d'évitement des catégories sociales, il est possible de remplacer les quatre catégories de l'administration scolaire par des catégories socioprofessionnelles plus détaillées. En écartant de l'analyse les sous-populations dont l'effectif trop faible dans Paris compromettrait la significativité des résultats (parents d'élèves agriculteurs et retraités), on dispose de vingt-quatre PCS. Il est possible de déterminer la probabilité d'éviter pour l'ensemble de chacune des catégories considérée, sa "structure par âge" (proportion d'élèves en avance, à l'heure, en retard), ainsi que la probabilité d'éviter dans chaque tranche d'âge (tableau 3, annexe 3). Une analyse en composantes principales est ainsi réalisée en prenant soin d'introduire la proportion d'élèves "à l'heure" et le taux d'évitement global sous forme de variables supplémentaires pour éviter la redondance des variables (tableau 4, annexe 4). Les deux premiers axes rendant compte des trois quarts de l'inertie totale (respectivement $45 \%$ et $30 \%$ ), il est possible de les retenir seuls et, du fait du petit nombre de variables, d'interpréter directement le plan factoriel.
\end{abstract}


Le premier axe est structuré en fonction du volume de capital scolaire possédé par les différentes catégories intégrées dans l'analyse. À l'extrémité de l'axe 1, du côté positif, on trouve les catégories socioprofessionnelles recourant le plus à l'évitement, au premier rang desquelles les enseignants et les instituteurs, qui possèdent le capital scolaire le plus important. Elles s'opposent ainsi aux catégories sociales qui, marquées par un plus fort retard scolaire, sont celles qui évitent le moins: du côté négatif de l'axe 1, on retrouve ainsi les catégories "moyennes" et "défavorisées" pour lesquelles éviter semble plus difficile. La structuration de cet axe permet de rendre compte de l'évitement lié au fait d'être "à l'heure" scolairement et, dans une moindre mesure, "en avance". Il exprime ainsi la plus ou moins grande dépendance des groupes sociaux envers l'école dans leurs stratégies de reproduction sociale.

Le second axe permet de rendre compte de l'opposition entre l'évitement selon le retard et l'avance scolaires. Il sépare du côté positif les catégories pour lesquelles il faut absolument être sinon "en avance", au moins "à l'heure" pour bénéficier d'un avantage en matière d'évitement ("instituteurs" surtout, mais aussi "cadres de la fonction publique") et, du côté négatif, les "professeurs et assimilés", pour lesquels le fait d'être "en avance" ne constitue plus un avantage déterminant pour éviter et qui peuvent mobiliser des ressources supplémentaires, en termes de capital culturel, social ou économique. Bien qu'élevée, la fréquence de l'avance n'est pas suffisante pour la banaliser. Pour des catégories comme les professeurs ou les "professions de l'information, de l'art ou du spectacle", l'âge de l'élève semble donc influer notablement moins sur les pratiques d'évitement que pour les autres catégories. Quant aux autres professions favorisées caractérisées par un capital économique important, leur positionnement est varié : les cadres d'entreprises et chefs d'entreprise reproduisent, à un degré moindre, les spécificités des instituteurs, tandis que les ingénieurs suivent plutôt la voie des professeurs. Les professions libérales semblent quant à elles développer un modèle intermédiaire. Chez les techniciens et employés de la fonction publique, la fréquence de l'avance scolaire et celle de l'évitement sont beaucoup plus modestes, ce qui leur vaut une coordonnée faiblement positive sur le premier axe. Pour eux, à l'inverse de ce que l'on a vu pour les professeurs, l'avance scolaire est un avantage certain pour éviter et le retard un grave handicap. Pour les techniciens en particulier, la variable de retard scolaire est particulièrement significative : sur cent enfants de techniciens en retard, moins de quinze parviennent à éviter. Tout se passe comme si les établissements désirés ne satisfaisaient, au sein de ces catégories, que les demandes d'évitement des élèves les plus "méritants".

Du côté des élèves de catégorie "moyenne" et "défavorisée", le deuxième axe introduit une coupure notable: les contremaîtres, employés, commerçants, artisans, policiers et autres professions intermédiaires sont caractérisés par des taux d'évitement particulièrement faibles, en particulier parmi les 
élèves "à l'heure". L'origine sociale semble bien ici un handicap pour éviter, handicap qui n'est que fort peu compensé par l'avance de l'élève. Seuls les artisans et professions intermédiaires de santé parviennent à éviter un peu plus souvent quand ils sont "en avance". Du côté positif de l'axe 2, on trouve les catégories les plus "défavorisées", marquées non seulement par un faible évitement, mais encore par un retard scolaire important. Toutefois, au sein même de ce quadrant du plan factoriel, la coordonnée sur le deuxième axe joue un rôle discriminant. Les PCS pour lesquelles cette coordonnée est faible (ouvriers qualifiés, inactifs et personnels de service), s'ils sont désavantagés dans la course à l'évitement, jouent néanmoins le jeu selon les mêmes règles que les autres catégories: être "en retard" les gêne pour éviter. Il n'est va pas de même des chômeurs et des ouvriers non qualifiés que leur coordonnée élevée sur l'axe 2 isole dans le plan factoriel. Ce qui les distingue n'est pas tant la fréquence du retard, trait qu'elles partagent avec les PCS précédentes, que l'association entre ce niveau de retard élevé et un mécanisme qui s'apparente à une prime au retard pour l'évitement. Dans ces catégories, non seulement les élèves "en retard" évitent plus souvent que les autres, mais encore le fait d'être "en avance" apparaît paradoxalement comme une un frein à l'évitement. Ce résultat peut s'interpréter comme un type d'évitement sous contraintes, lié aux politiques de sélection scolaire des établissements qui refusent la réinscription d'élèves en retard, ou ne leur permettent pas de redoubler dans les sections demandées faute de place.

\section{Conclusion}

G $\mathrm{n}$ définitive, l'analyse des mécanismes d'évitement scolaire semble expri-

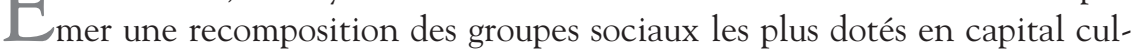
turel et de leurs stratégies de reproduction dans leur relation à l'école (comme l'indique notamment l'axe 2 du précédent modèle). Plusieurs facteurs permettent de soutenir cette interprétation, et notamment les caractéristiques différentes de l'évitement des catégories "instituteurs" et "cadres de la fonction publique" d'une part, des catégories "professeurs" et "professions de l'information, de l'art et du spectacle" d'autre part. L'accroissement de la concurrence scolaire a pour effet une diffusion des pratiques d'évitement qui affecte leur rentabilité scolaire et place les catégories sociales dont le statut dépend le plus de la possession de capital scolaire dans l'obligation de transformer leurs stratégies de reproduction. En matière de pratiques scolaires comme ailleurs, les catégories les mieux dotées sont aussi les plus aptes à reconvertir le cas échéant leurs investissements scolaires hors des filières scolaires les plus valorisées et les plus prestigieuses. Tout se passe comme si on assistait à la diffusion vers l'enseignement secondaire de mécanismes déjà observés au 
cours des années 1970 dans les stratégies de reconversion des classes dominantes au niveau des filières de l'enseignement supérieur, en particulier vers les "professions culturelles" favorisant la rentabilisation de l'héritage familial non médiatisé par l'école, ces filières servant ainsi de refuge aux enfants de la classe dominante que l'école a éliminés (Bourdieu 1979, 145-188). Ce déplacement est alors susceptible d'expliquer que les catégories sociales les plus favorisées utilisent, moins qu'on ne pourrait s'y attendre, des formes d'évitement liées à l'âge de l'élève, au profit de stratégies plus rentables et, pour certaines, moins diffusées: recours à l'enseignement privé, mais surtout évitement précoce, dès l'école primaire, voire la fin de la maternelle, ce qui implique l'incorporation des choix résidentiels dans les stratégies scolaires. Mais dans l'un ou l'autre cas, ces transformations supposent une revalorisation du capital économique comme principe de différenciation des pratiques scolaires et de leur rentabilisation. Face à cette dévaluation du capital scolaire et de sa rentabilité, les familles dont la reproduction dépend le plus du capital scolaire tout en se trouvant moins bien dotées en autres formes de capitaux ("instituteurs", "cadres de la fonction publique", etc.) se trouvent confrontées, en fonction de leurs secteurs de résidence et des politiques rectorales mises en place, non seulement à une restriction de leurs possibles scolaires liée à l'intensification de la concurrence, mais aussi à des difficultés croissantes pour réaliser les investissements scolaires les plus rentables, dont l'évitement constitue un élément important.

Une autre hypothèse interprétative sur laquelle débouche donc cette modélisation statistique est l'importance croissante des facteurs spatiaux: la localisation géographique, liée au prix du logement intervient comme facteur intermédiaire dans la revalorisation du capital économique pour les stratégies de scolarisation, notamment par le biais des "choix" résidentiels offerts aux différentes catégories sociales (prix des loyers ou du mètre carré dans l'accès à la propriété). Cet effet de lieu implique qu'il n'y ait pas une répartition socialement aléatoire de la distribution spatiale, mais une répercussion sur le type d'établissement possible autour du lieu de résidence. Une étape supplémentaire de ce travail consistera donc à prendre en compte la localisation des élèves et à faire intervenir les variables de distance spatiale. Cette distance influence d'une part l'accessibilité physique aux établissements et, d'autre part, l'accessibilité à l'information —celle-ci étant liée à la distance spatiale, même si les réseaux sociaux peuvent la compenser en partie. De ce point de vue les classes moyennes, qui sont plus proches, géographiquement, des milieux populaires, subissent avec elles la pesanteur de leur localisation, tandis que les catégories favorisées, qui sont déjà avantagées par leur localisation géographique, sont en outre capables de s'affranchir des "nécessités" spatiales. 


\section{Bibliographie}

BERSTEIN S. 2001 Préface à RuHLmanN J., Ni bourgeois ni prolétaires. La défense des classes moyennes en France au XXe siècle, Paris, Seuil

BOURDIEU P. 1979 La distinction, Paris, Minuit

BROCCOLICHI S. \& van ZANTEN A. 1997 "Espaces de concurrence et circuits de scolarisation. L'évitement des collèges publics d'un district de la banlieue parisienne", Annales de la recherche urbaine, 75 et suivantes

FRANÇOIS J.-C. 2002 "Évitement à l'entrée en sixième et division sociale de l'espace scolaire à Paris", L'Espace Géographique, 4, 307-327

FRANÇOIS J.-C., MATHIAN H., RIBARDIÈRE A. \& SAINT-JULIEN T. 2002 Les disparités des revenus des ménages franciliens en 1999. Approches intercommunale/infracommunale et évolution des différenciations intercommunales (1990-1999), UMR Géographie Cités

LANGOUËT G. \& LÉGER A. 1997 Le choix des familles, École publique ou école privée, Paris, Faber

POUPEAU F. 2004, Contestations scolaires et ordre social. Les enseignants de Seine-SaintDenis en grève, Paris, Syllepse

\section{Annexe 1}

La procédure de dérogation, établie par le ministère, est en principe commune à tous les départements français. Cependant, la relative autonomie dont jouissent les inspecteurs d'académie en la matière entraîne des nuances importantes, non seulement dans la proportion des décisions favorables, mais encore dans l'organisation de la procédure ellemême. La présentation du dossier de demande de dérogation rempli par la famille peut varier sensiblement d'un département à l'autre. À Paris, onze motifs sont proposés sur le formulaire de dérogation: santé, langue vivante, fratrie dans le collège, parents travaillant dans l'établissement, proximité du domicile, horaires aménagés, établissement "international", fratrie dans le lycée, proximité du travail des parents, hébergement dans le secteur de l'établissement désiré, autres.

Pour plus de détails sur le phénomène d'évitement, voir la réponse à l'appel d'offres sur "Les disparités territoriales", pour le compte du ministère de l'Éducation nationale, du ministère de la Recherche et de la DATAR: Champagne P., François J.-C., Poupeau F. \& Rhein C. Les trajectoires de l'évitement. Une analyse sociologique et géographique des flux d'élèves dans le système scolaire francilien. 


\section{Annexe 2}

\section{Le modèle logit}

Ce modèle peut s'écrire sous la forme suivante:

\section{Évitement $=$ cste + a.PCS + b.AGE + c.PCS*ÂAG}

II s'agit d'un modèle "saturé" dans lequel figurent les interactions entre variables. En effet, une fois que les variables PCS et ÂGE ont toutes deux épuisé leur pouvoir explicatif propre, il demeure possible que l'interaction des deux (PCS*ÂGE) puisse jouer un rôle significatif en ayant elle-même un pouvoir explicatif supplémentaire. Le critère de convergence retenu est celui du maximum de vraisemblance. Un élève est en situation d'évitement (évitement $=\mathrm{I}$ ) quand, résidant à Paris intra-muros, il est scolarisé dans un collège public différent de son collège de secteur (les élèves scolarisés dans l'enseignement privé ne sont pas pris en compte).

Les trois composantes explicatives sont significatives, mais avec des nuances. La variable ÂGE est de loin la plus significative, avec un risque d'erreur très faible (alpha < // 10000). L'évitement se ferait-il avant tout "au mérite" ? Ce n'est pas surprenant si l'on songe que le choix d'une option rare est le motif de dérogation le plus fréquent. Une partie du pouvoir explicatif de l'appartenance à une catégorie sociale est déjà neutralisée par cette variable ÂGE, puisque les catégories sociales se distinguent les unes les autres par leur structure par âge. II n'est donc pas étonnant que le pouvoir explicatif de la variable PCS soit plus modeste; la significativité de celle-ci reste néanmoins acceptable (risque d'erreur alpha $=\mathrm{l} / \mathrm{I} 0$ ). II demeure donc une inégalité face à l'évitement entre élèves des différentes catégories sociales, toutes choses égales quant à leur âge. Mais cette "inégalité sociale" face à l'évitement s'exprime aussi, et bien davantage, par l'appartenance croisée de l'élève à une classe d'âge et une catégorie sociale. Le bon niveau de significativité de l'interaction ÂGE*PCS (alpha<5//00) montre que le croisement de ces deux appartenances possède un important pouvoir explicatif supplémentaire, toutes choses égales quant aux variables ÂGE et PCS.

\section{Annexe 3}

Tableau 3

Relation entre l'âge de l'élève, la PCS et l'évitement (élèves de nationalité française)

\begin{tabular}{|c|c|c|c|c|c|}
\hline Catégories socioprofessionnelles (PCS) & $\begin{array}{l}\text { Taux global } \\
\text { d'évitement } \\
\text { dans la PCS }\end{array}$ & Âge & $\begin{array}{c}\text { Structure } \\
\text { par âge } \\
\text { de la PCS }\end{array}$ & $\begin{array}{c}\text { Probabilité } \\
\text { d'éviter } \\
\text { dans } \\
\text { chaque } \\
\text { classe d'âge }\end{array}$ & \begin{tabular}{|c|} 
Signe du \\
coefficient \\
correspondant \\
dans le \\
modèle
\end{tabular} \\
\hline Artisans & 0,28 & $\begin{array}{l}\text { avance } \\
\text { à l'heure } \\
\text { retard }\end{array}$ & $\begin{array}{r}3,9 \\
72,9 \\
23,2\end{array}$ & $\begin{array}{l}0,41 \\
0,41 \\
0,17\end{array}$ & $\begin{array}{l}+ \\
+ \\
-\end{array}$ \\
\hline Inactifs & 0,32 & $\begin{array}{l}\text { avance } \\
\text { à l'heure } \\
\text { retard }\end{array}$ & $\begin{array}{r}2,4 \\
54 \\
43,6\end{array}$ & $\begin{array}{l}0,42 \\
0,34 \\
0,29\end{array}$ & $\begin{array}{l}\text { (ns) } \\
\text { (ns) } \\
\text { (ns) }\end{array}$ \\
\hline Cadres d'entreprise & 0,38 & $\begin{array}{l}\text { avance } \\
\text { à l'heure } \\
\text { retard }\end{array}$ & $\begin{array}{r}9,6 \\
82,6 \\
7,8\end{array}$ & $\begin{array}{r}0,38 \\
0,4 \\
0,28\end{array}$ & $\begin{array}{l}\text { (ns) } \\
\text { (ns) } \\
\text { (ns) }\end{array}$ \\
\hline Cadres de la fonction publique & 0,41 & $\begin{array}{l}\text { avance } \\
\text { à l'heure } \\
\text { retard }\end{array}$ & $\begin{array}{r}11,6 \\
79,4 \\
9\end{array}$ & $\begin{array}{r}0,46 \\
0,4 \\
0,32\end{array}$ & $\begin{array}{l}\text { (ns) } \\
\text { (ns) } \\
\text { (ns) }\end{array}$ \\
\hline Chefs d'entreprise & 0,38 & $\begin{array}{l}\text { avance } \\
\text { à l'heure } \\
\text { retard }\end{array}$ & $\begin{array}{r}8,6 \\
85,6 \\
5,8\end{array}$ & $\begin{array}{r}0,4 \\
0,39 \\
0,3\end{array}$ & $\begin{array}{l}\text { (ns) } \\
\text { (ns) } \\
\text { (ns) }\end{array}$ \\
\hline
\end{tabular}




\begin{tabular}{|c|c|c|c|c|c|}
\hline Catégories socioprofessionnelles (PCS) & $\begin{array}{l}\text { Taux global } \\
\text { d'évitement } \\
\text { dans la PCS }\end{array}$ & Âge & $\begin{array}{c}\text { Structure } \\
\text { par âge } \\
\text { de la PCS }\end{array}$ & $\begin{array}{c}\text { Probabilité } \\
\text { d'éviter } \\
\text { dans } \\
\text { chaque } \\
\text { classe d'âge }\end{array}$ & $\begin{array}{c}\text { Signe du } \\
\text { coefficient } \\
\text { correspondant } \\
\text { dans le } \\
\text { modèle }\end{array}$ \\
\hline \multirow{2}{*}{ Chômeurs cherchant un premier emploi } & 0,36 & avance & 2 & 0 & (ns) \\
\hline & & à l'heure & 49,7 & $\begin{array}{r}0,4 \\
033\end{array}$ & (ns) \\
\hline \multirow{2}{*}{ Commerçants } & 0,3 & $\begin{array}{l}\text { retard } \\
\text { avance }\end{array}$ & $\begin{array}{r}48,3 \\
3,9\end{array}$ & $\begin{array}{l}0,33 \\
0,21\end{array}$ & $\begin{array}{c}(\mathrm{ns}) \\
\text { (ns) }\end{array}$ \\
\hline & & à l'heure & $\begin{array}{l}75,3 \\
198\end{array}$ & 0,33 & + \\
\hline \multirow[t]{2}{*}{ Contremaîtres agents de maîtrise } & 0,27 & $\begin{array}{l}\text { avance } \\
\text { à l'heure }\end{array}$ & $\begin{array}{r}3,2 \\
81,7\end{array}$ & $\begin{array}{l}0,66 \\
0,26\end{array}$ & $\begin{array}{l}\text { (ns) } \\
\text { (ns) }\end{array}$ \\
\hline & & retard & 15,1 & 0,21 & (ns) \\
\hline \multirow[t]{2}{*}{ Employés d'entreprise } & 0,32 & $\begin{array}{l}\text { avance } \\
\text { à l'heure }\end{array}$ & $\begin{array}{r}1,8 \\
73,7\end{array}$ & $\begin{array}{l}0,38 \\
0,35\end{array}$ & $\begin{array}{l}\text { (ns) } \\
\text { (ns) }\end{array}$ \\
\hline & & retard & 24,5 & 0,19 & (in) \\
\hline \multirow[t]{2}{*}{ Employés de commerce } & 0,33 & avance & 2 & 0,57 & (ns) \\
\hline & & $\begin{array}{l}\text { à l'heure } \\
\text { retard }\end{array}$ & $\begin{array}{l}69,2 \\
28,8\end{array}$ & $\begin{array}{r}0,33 \\
0,3\end{array}$ & $\begin{array}{l}(\mathrm{ns}) \\
\text { (ns) }\end{array}$ \\
\hline \multirow[t]{2}{*}{ Employés de la fonction publique } & 0,28 & avance & $\begin{array}{l}11,6 \\
79,3\end{array}$ & $\begin{array}{l}0,25 \\
0,33\end{array}$ & $\begin{array}{l}\text { (ns) } \\
\text { (ns) }\end{array}$ \\
\hline & & retard & 9,1 & 0,19 & (ns) \\
\hline \multirow[t]{2}{*}{ Ingénieurs } & 0,36 & avance & 11,6 & 0,38 & (ns) \\
\hline & & $\begin{array}{l}\text { à l'heure } \\
\text { retard }\end{array}$ & $\begin{array}{r}83,1 \\
5,3\end{array}$ & $\begin{array}{l}0,37 \\
0,21\end{array}$ & $\begin{array}{l}\text { (ns) } \\
\text { (ns) }\end{array}$ \\
\hline \multirow[t]{2}{*}{ Intituteur } & 0,47 & $\begin{array}{l}\text { avance } \\
\text { à l'heure }\end{array}$ & $\begin{array}{r}8,5 \\
85\end{array}$ & $\begin{array}{l}0,47 \\
0,49\end{array}$ & (ns) \\
\hline & & $\begin{array}{l}\text { a neure } \\
\text { retard }\end{array}$ & 6,5 & 0,31 & (ns) \\
\hline \multirow[t]{2}{*}{ Ouvrier non qualifié } & 0,34 & $\begin{array}{l}\text { avance } \\
\text { à l'heure }\end{array}$ & $\begin{array}{l}0,5 \\
52\end{array}$ & $\begin{array}{r}0,33 \\
0,4\end{array}$ & $\begin{array}{l}\text { (ns) } \\
\text { (ns) }\end{array}$ \\
\hline & & $\begin{array}{l}\text { à lheure } \\
\text { retard }\end{array}$ & $\begin{array}{r}32 \\
47,5\end{array}$ & 0,41 & $\begin{array}{l}\text { (ns) } \\
\text { (ns) }\end{array}$ \\
\hline \multirow[t]{3}{*}{ Ouvrier qualifié } & 0,29 & avance & 1,5 & 0,21 & (ns) \\
\hline & & à l'heure & 58,5 & 0,32 & (ns) \\
\hline & & retard & 40 & 0,22 & (ns) \\
\hline \multirow[t]{2}{*}{ Personnel de service aux particuliers } & 0,31 & avance & 1,7 & 0,33 & (ns) \\
\hline & & à l'heure & 61,7 & $\begin{array}{l}0,33 \\
0,25\end{array}$ & (ns) \\
\hline \multirow[t]{2}{*}{ Policier, militaire } & 0,32 & avance & I,5 & 0,5 & (ns) \\
\hline & & à l'heure & 78,8 & $\begin{array}{l}0,34 \\
0,23\end{array}$ & $\begin{array}{l}(\mathrm{ns}) \\
\text { (ns) }\end{array}$ \\
\hline \multirow{3}{*}{ Professeurs et assimilés } & 0,41 & $\begin{array}{l}\text { retard } \\
\text { avance }\end{array}$ & $\begin{array}{l}19,1 \\
128\end{array}$ & 0,47 & $\begin{array}{ll}(1 \mathrm{I}) \\
+\end{array}$ \\
\hline & & à l'heure & 82,5 & 0,41 & + \\
\hline & & retard & 4,7 & 0,16 & - \\
\hline \multirow[t]{2}{*}{ Professions information, arts, spectacle } & 0,39 & avance & 8,6 & 0,41 & + \\
\hline & & à l'heure & $\begin{array}{r}83,4 \\
8\end{array}$ & $\begin{array}{l}0,41 \\
0,17\end{array}$ & + \\
\hline \multirow[t]{2}{*}{ Professions intermédiaires (commerce) } & 0,34 & avance & 5,2 & 0,42 & (ns) \\
\hline & & à l'heure & 82,3 & 0,34 & (ns) \\
\hline \multirow[t]{3}{*}{ Professions intermédiaires (santé) } & 0,31 & $\begin{array}{l}\text { retard } \\
\text { avance }\end{array}$ & $\begin{array}{r}2,5 \\
2,8\end{array}$ & $\begin{array}{l}0,2 \\
0,6\end{array}$ & + \\
\hline & & à l'heure & 72 & 0,32 & (ns) \\
\hline & 0.35 & retard & $\begin{array}{r}25,2 \\
5,4\end{array}$ & $\begin{array}{r}0,23 \\
0,5\end{array}$ & (ns) \\
\hline \multirow{2}{*}{ Professions intermédiaires (fonction publique) } & 0,03 & à l'heure & 76 & 0,35 & (ns) \\
\hline & & retard & 18,6 & 0,29 & (ns) \\
\hline \multirow[t]{3}{*}{ Professions libérales } & 0,39 & avance & 9,7 & 0,42 & (ns) \\
\hline & & à l'heure & 84,8 & 0,39 & (ns) \\
\hline & & retard & 5,5 & 0,26 & (ns) \\
\hline Techniciens & 0,32 & avance & 5,8 & 0,5 & (ns) \\
\hline & & à l'heure & $\begin{array}{r}74,1 \\
5\end{array}$ & $\begin{array}{l}0,36 \\
014\end{array}$ & (ns) \\
\hline
\end{tabular}




\section{Annexe 4}

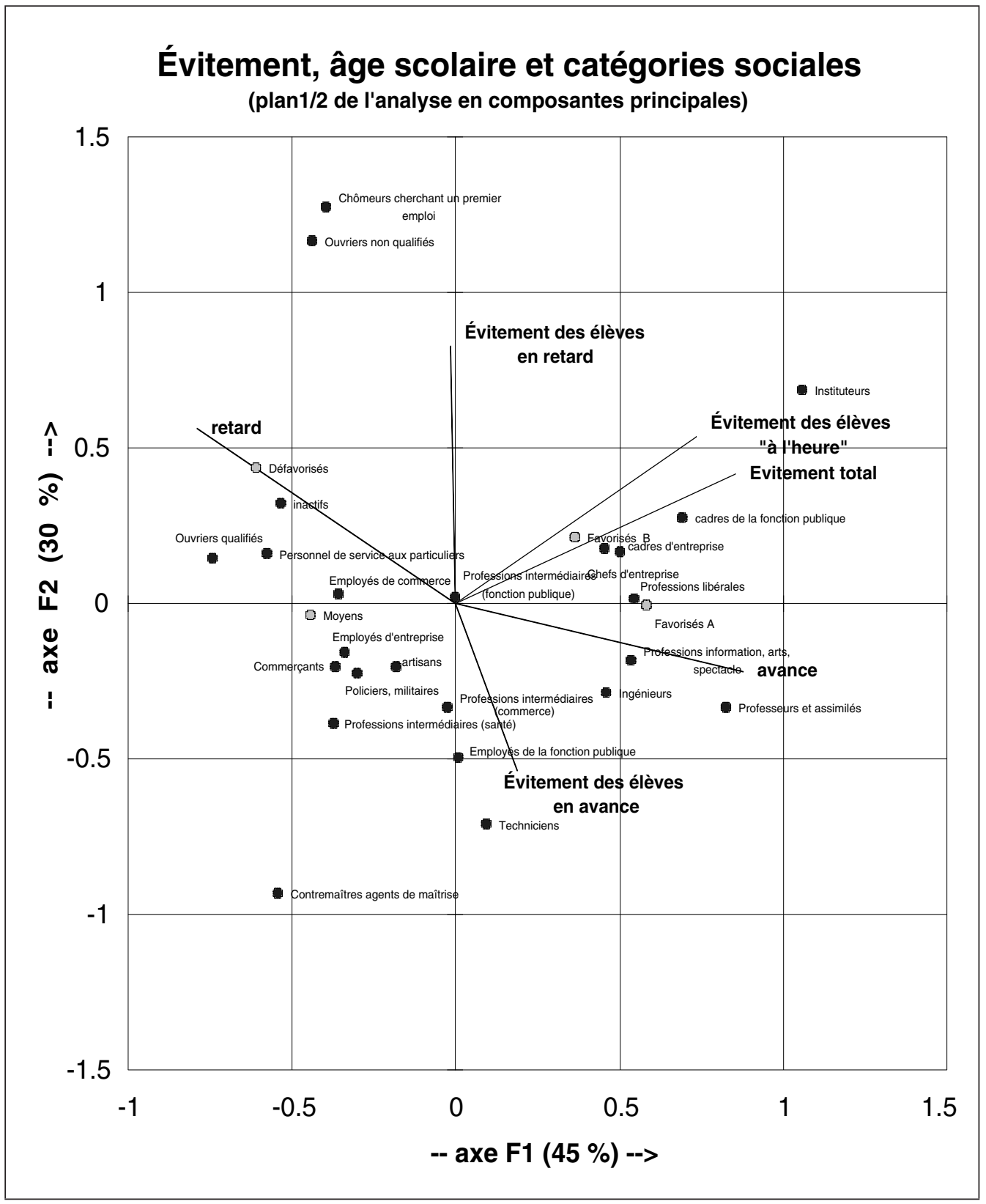

